\title{
Hydrophilic PDMS microchannels for high-throughput formation of oil-in-water microdroplets and water-in-oil-in-water double emulsions $\dagger$
}

\author{
Wolfgang-Andreas C. Bauer, ${ }^{a}$ Martin Fischlechner, ${ }^{b}$ Chris Abell ${ }^{b}$ and Wilhelm T. S. Huck *ac \\ Received 9th March 2010, Accepted 15th April 2010 \\ First published as an Advance Article on the web 5th May 2010 \\ DOI: 10.1039/c004046k
}

Here we present a novel surface modification method based on the sequential layer-by-layer deposition of polyelectrolytes yielding hydrophilic microchannels in PDMS-based microfluidic devices. The coatings are long-term stable and allow for the generation of monodisperse oil-in-water microdroplets even several months after the channel surface treatment. Due to the robustness of the polyelectrolyte multilayers ultra-high flow rates can be applied, making high-throughput droplet formation in the jetting mode possible. Furthermore, we successfully used our method to selectively modify the surface properties in certain areas of assembled microchannels. The resulting partially hydrophilic, partially hydrophobic microfluidic devices allow for the production of monodisperse water-in-oil-in-water double emulsions.

\section{Introduction}

Poly(dimethylsiloxane) (PDMS) has become one of the most commonly used materials in microfluidic device fabrication. ${ }^{1}$ Compared to silicon and glass devices, PDMS-based chips can be manufactured much faster, easier and cheaper by means of soft lithography. ${ }^{2}$ Furthermore, PDMS can be cured at low temperature, is optically transparent, biologically inert, non-toxic and non-flammable as well as permeable to gases. It also seals with numerous other materials enabling the fabrication of hybrid microfluidic devices. ${ }^{3}$ However, due to its hydrophobicity PDMS encounters significant limitations when stable oil-in-water $(\mathrm{o} / \mathrm{w})$ emulsions are to be produced. The microchannel surface must be turned hydrophilic to assure an effective wetting of the channel walls by the continuous aqueous solution. The various surface modification methods ${ }^{3,4}$ found in literature have significant drawbacks:

(i) Ionic wetting agents can be added to the aqueous phase for a dynamic surface modification. ${ }^{5}$ However, they also influence droplet formation and stability and can have unfavorable interactions with other surface active compounds present in the system.

(ii) Oxidation of the PDMS surface, for example by oxygen plasma, ${ }^{6}$ only leads to temporarily hydrophilic surfaces as PDMS regains its original surface properties over time. ${ }^{7}$ Storage of the oxidized devices in a high-surface-energy medium like water can postpone this hydrophobic recovery, but it inevitably sets in as soon as the surface is exposed to a low-surface-energy medium such as air. ${ }^{8}$

\footnotetext{
${ }^{a}$ Melville Laboratory for Polymer Synthesis, Department of Chemistry, University of Cambridge, Lensfield Road, Cambridge, UK CB2 1EW. E-mail:wtsh2@cam.ac.uk; Tel: +44(0)1223 334370

${ }^{b}$ Department of Chemistry, University of Cambridge, Lensfield Road, Cambridge, UK CB2 1EW

${ }^{c}$ Radboud University Nijmegen, Institute for Molecules and Materials, Heyendaalseweg 135, 6525 AJ Nijmegen, The Netherlands

$\dagger$ Electronic supplementary information (ESI) available: Videos and experimental details. See DOI: 10.1039/c004046k
}

(iii) Chemical vapour deposition can create permanent coatings, ${ }^{9}$ but is limited to non-assembled chips as unhindered access of the vapour to the substrate is mandatory. Device fusion, typically involving plasma oxidation, can destroy the coating afterwards.

(iv) Covalent surface modification via graft photopolymerization ${ }^{10}$ can be challenging in assembled PDMS channels as the reaction tends to occur in the channel lumen rather than at the walls. Allbritton et al. ${ }^{11}$ solved this problem by preadsorption of a photoinitiator prior to polymerization. However, both the photoinitiator and the monomer solution are manually injected into the chip, and multiple washings are required, making the procedure rather elaborate.

(v) Weitz et al. ${ }^{12}$ use sol-gel methods to modify PDMS permanently with a glass coating, which is robust against organic solvents and can be tailored in its properties. But coating thicknesses of up to $10 \mu \mathrm{m}$ strongly distort channel dimensions and interfere with complex device geometries. Besides, a reaction time in the range of a few seconds for the key gelation reaction makes the procedure rather delicate to handle.

(vi) Layer-by-layer (LbL) deposition of positively and negatively charged species onto a substrate, a technique introduced by Decher et al., ${ }^{13}$ has been successfully used to modify the inner surface of capillaries and microchannels. ${ }^{14}$ This method usually follows straightforward protocols and offers an immense versatility. However, existing procedures involve the manual injection and removal of solutions both for layer formation and washing steps. Furthermore, deposition times are typically in the range of several minutes per layer, which makes the throughput rate for PDMS chip modification relatively low.

\section{Results and discussion}

We developed the LbL approach further and invented a largely automated method producing long-term stable hydrophilic coatings onto PDMS microchannels (Fig. 1). In Fig. 1(a) the schematics of the process are shown: the key feature is the loading of a piece of tubing with solution segments, all separated 

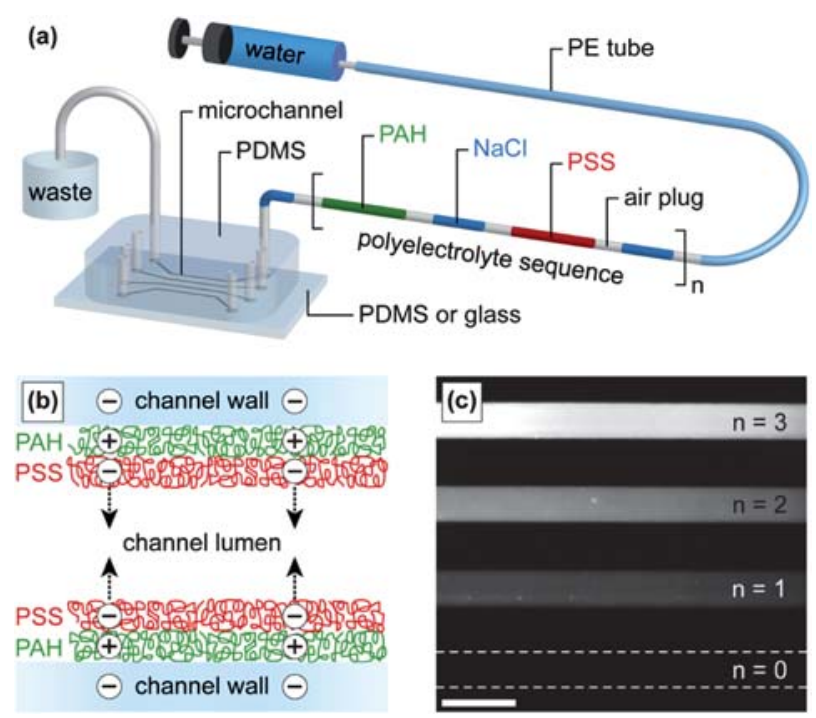

Fig. 1 (a) Schematic of the LbL surface modification of a PDMS microchannel. Segments of $\mathrm{NaCl}, \mathrm{PAH}$ and PSS solutions, separated by air plugs, are loaded into a piece of tubing and sequentially flushed through the channel at a constant flow rate. (b) Schematic of a PEM building up at the channel wall. (c) Fluorescence micrograph of four microchannels modified with different solution sequences $(0 \leq n \leq 3)$. The fluorescence intensity rises with increasing $n$. Scale bar denotes $100 \mu \mathrm{m}$.

by air plugs. ${ }^{15} \mathrm{~A}$ syringe is used to draw into the tube alternately segments of poly(allylamine hydrochloride) (PAH) and poly(sodium 4-styrenesulfonate) (PSS) solutions (both $0.1 \% \mathrm{w} / \mathrm{v}$ in $0.5 \mathrm{M}$ aqueous $\mathrm{NaCl}$ solution) with aqueous $\mathrm{NaCl}$ washing solution $(0.1 \mathrm{M})$ segments in between. This concept is very versatile and allows for a fine-tuning of the channel surface properties since all imaginable combinations of solutions can be applied. The loading of the tubing with the desired number of segments is the only task within the procedure which is carried out manually, but 10 segments can easily be drawn in within five minutes. Subsequently, the tubing is connected to the microfluidic chip directly after plasma treatment and assembly, when the channel walls are still hydrophilic. Using a syringe pump the segments successively enter the microchannel, flush it and are blown out again by the following air plug. The residence time of each solution inside the microchannel can be precisely adjusted by the flow rate and the length of the respective segment. Following existing LbL surface coating protocols ${ }^{13,14}$ we ensured an absorption time for the polyelectrolytes of several minutes per layer. The final washing step is performed with deionized water (DIW) in order to remove traces of salt from the channel walls.

Fig. 1(b) shows schematically the polyelectrolyte multilayer (PEM) that self-assembles at the channel wall. The first PAH solution segment enters the channel when negative charges created during the plasma treatment are still present at the PDMS surface and a layer of the positively charged polyelectrolyte is deposited. The overcompensation ${ }^{13}$ of the surface charge by the incoming PAH layer leads to a positively charged surface after this step. PAH polycations that are not firmly bound to the channel wall are removed by the following $\mathrm{NaCl}$ solution segment. The PSS polyanions flushed through the channel in the subsequent plug can deposit onto the PAH layer causing again an overcompensation of the charge and another sign reversal at the interface. After rinsing the channel with washing solution the next PAH layer can be applied and so on.

We analyzed the resultant coating via fluorescence microscopy using a solution of fluorescently labeled PAH $(0.1 \% \mathrm{w} / \mathrm{v}$ in $0.5 \mathrm{M}$ aqueous $\mathrm{NaCl}$ solution). The micrograph in Fig. 1(c) shows the central part of a microfluidic chip with both bottom and top surface made of PDMS. Four microchannels were independently modified applying our plug-based method. Different solution sequences with varying numbers $n$ of PAH segments $(0 \leq n \leq 3)$ were flushed through the channels at a constant flow rate of $50 \mu \mathrm{L} \mathrm{h}^{-1}$. The fluorescence analysis revealed that for the negative control $(n=0)$ no measurable fluorescence signal above background could be detected while for the other channels an increase in fluorescence intensity with increasing $n$ was observed. These results show that our method is not only convenient and time-saving, but that the process of gradual multilayer assembly is also well-controlled.

\section{Long-term stability of the hydrophilic coating}

Having established our surface modification technique we tested the durability of the applied hydrophilic coatings. Microfluidic devices coated with a PAH-PSS-PAH-PSS PEM were kept at room temperature (rt) in ambient air. After different storage times we used these devices for the production of hexadecane droplets in DIW containing $0.5 \% \mathrm{w} / \mathrm{w}$ of the surfactant sodium dodecylsulfate (SDS). Lowering the interfacial tension between oil and water this compound promotes a smooth droplet formation and increases droplet stability against coalescence.

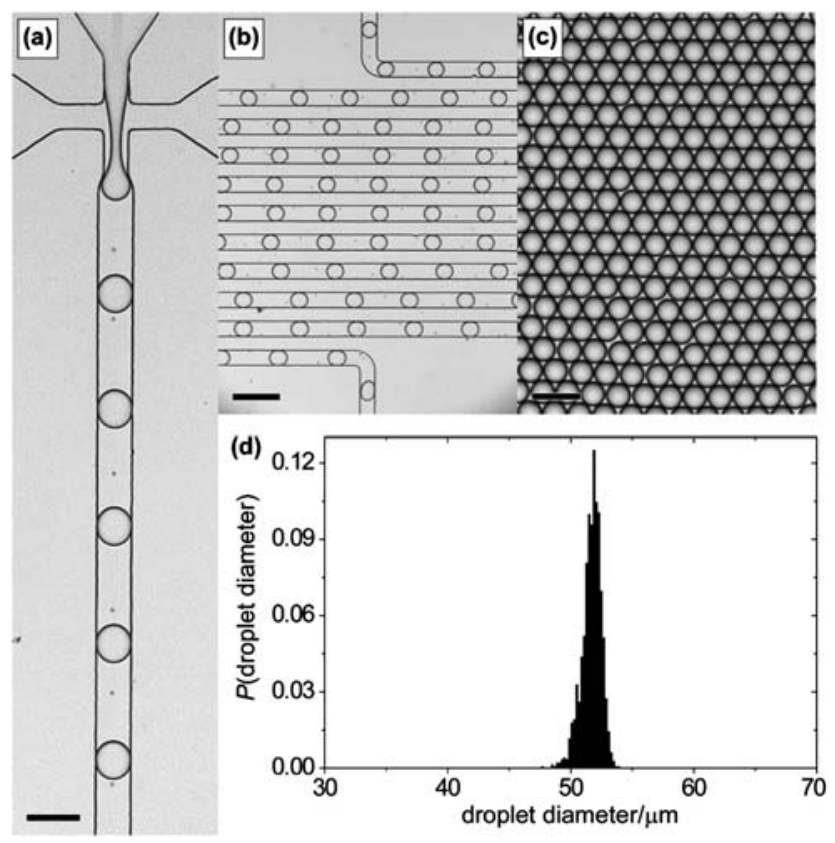

Fig. 2 Production of hexadecane-in-water droplets using a modified device after more than 5 months of storage. Micrographs of (a) the stable formation of droplets and (b) their passage through the chip prove that the channel walls are still hydrophilic. (c) This micrograph depicts the hexagonal close-packed monolayer adopted by the droplets in a storage device. (d) The diameter distribution shows a high level of droplet monodispersity. Scale bars denote (a) $100 \mu \mathrm{m}$, (b) $200 \mu \mathrm{m}$ and (c) $100 \mu \mathrm{m}$. 
Fig. 2 refers only to the longest storage time of more than 5 months between surface modification and droplet formation, but the results agree entirely with the other experiments in this experimental study.

In Fig. 2(a) the stable formation of uniform droplets occurring at a frequency of $170 \mathrm{~Hz}$ at the flow-focusing region ${ }^{16}$ is depicted. The flow rates of hexadecane and DIW were $50 \mu \mathrm{L} \mathrm{h}^{-1}$ and $150 \mu \mathrm{L} \mathrm{h}^{-1}$, respectively. During the observation period of $90 \mathrm{~min}$ no signs of degradation in hydrophilic surface properties were detected. The droplets travelled through the device without difficulty or wetting the walls (Fig. 2(b)).

A small amount (ca. $5 \mu \mathrm{L})$ of the emulsion was collected and injected into a storage device. Fig. 2(c) shows a micrograph of the hexagonal close-packed monolayer of droplets assembled inside the reservoir. To quantify the monodispersity of the droplets we determined the diameter distribution of 1463 droplets, as shown in Fig. 2(d). Droplets have a mean diameter of $51.7 \mu \mathrm{m}$ with a standard deviation of $0.8 \mu \mathrm{m}$ (about $1.5 \%$ ).

In this series of experiments we demonstrated that our method yields hydrophilic channel coatings exhibiting a remarkable longterm stability. Modified devices can be kept in stock over months and still allow for the production of monodisperse emulsions in a highly reproducible manner.

\section{High-throughput production of $\mathrm{o} / \mathrm{w}$ microdroplets}

In order to evaluate the robustness of coatings deposited via our method we performed an endurance test, i.e. the high-throughput production of large emulsion volumes. However, with increasing flow rates of the continuous and the dispersed phase in a coflowing system and, hence increasing capillary number, the formation of droplets shifts from the dripping mode towards jetting. This transition has been studied extensively. ${ }^{17}$ Within the jetting regime droplet generation does no longer occur at the flow-focusing region, but the jet breaks up further downstream due to the Rayleigh-Plateau instability. ${ }^{18}$ We built a microfluidic device exhibiting a wide cavity after the flow-focussing region and modified the microchannel with a PAH-PSS-PAH-PSS PEM. This design ${ }^{19}$ reportedly regularizes the jet breakup as perturbations of the flow field inside the chamber caused by droplets passing through the small exit channel feed back to the jet. In order to prevent leakage at high flow rates we glued the tubing in the channel using PDMS. To ensure complete curing, the device was kept at $\mathrm{rt}$ for one day prior to experiment; this would have been impossible without a long-term stable coating preserving the hydrophilic surface properties over time.

In the microfluidic experiment (Fig. 3) we used again hexadecane as dispersed phase and DIW containing $0.5 \% \mathrm{w} / \mathrm{w}$ SDS as continuous phase. Fig. 3(a) shows the formation of droplets at an oil flow rate of $1000 \mu \mathrm{L} \mathrm{h}^{-1}$ and a DIW flow rate of $4500 \mu \mathrm{L}$ $\mathrm{h}^{-1}$ by controlled jet breakup in the cavity. The droplet generation frequency was $13.5 \mathrm{kHz}$. Without any wetting problems the hexadecane droplets proceeded smoothly through the whole chip (Fig. 3(b)).

Parts of the collected emulsion sample were injected into a storage device. Fig. 3(c) shows the droplets assembled into a hexagonal close-packed monolayer. The droplet diameter distribution of 1020 droplets, given in Fig. 3(d), is extremely narrow and yields a mean value of $35.8 \mu \mathrm{m}$. As the standard
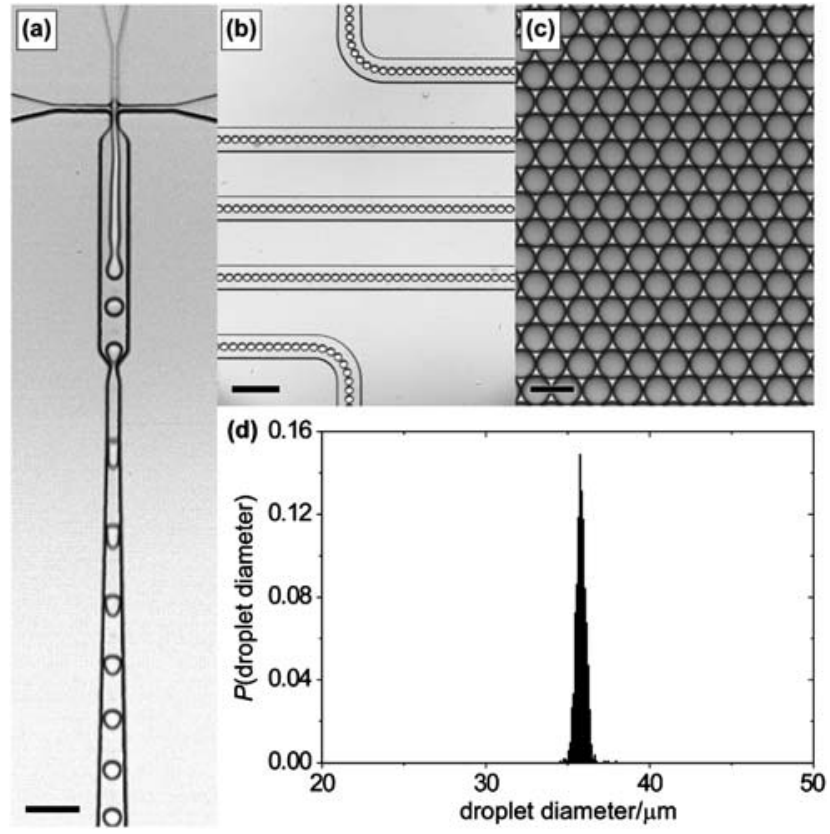

Fig. 3 High-throughput production of hexadecane-in-water droplets by regularized jet breakup. Micrographs of (a) the formation of droplets and (b) the passage of droplets through the chip without any wetting defects. (c) This micrograph shows the hexagonal close-packed monolayer adopted by the produced droplets in a storage reservoir. (d) The diameter distribution reveals a very high level of droplet monodispersity. Scale bars denote (a) $100 \mu \mathrm{m}$, (b) $200 \mu \mathrm{m}$ and (c) $50 \mu \mathrm{m}$.

deviation of $0.3 \mu \mathrm{m}$ is below $1 \%$ of the mean diameter the droplets can be considered highly monodisperse.

These results prove that our surface modification method is suitable for high-throughput droplet formation as it produces very robust hydrophilic coatings which can withstand even extremely high flow rates. Those operating conditions are desirable as large volumes of highly monodisperse droplets are of great importance, especially for the formation of polymer particles. ${ }^{20}$

\section{Production of w/o/w double emulsions}

Having successfully used our LbL deposition technique for the production of $\mathrm{o} / \mathrm{w}$ microdroplets we addressed the fabrication of more complex devices that can generate double emulsions. ${ }^{21,22}$ Especially w/o/w double emulsions are highly attractive systems as they have great potential for applications, for instance as potential delivery systems for pharmacological compounds. ${ }^{23} \mathrm{As}$ traditional bulk preparation methods ${ }^{24}$ usually yield w/o/w droplets with a broad size distribution a microfluidic approach is advantageous when monodisperse double emulsions are required. This is not trivial as two droplet production processes have to be precisely controlled. In order to engulf a defined number of small water droplets into the bigger oil droplets both formation frequencies have to be synchronized. This is done best when performing both processes on a single microfluidic chip. However, when using PDMS-based devices the wettability of the channel surface poses another challenge. For the formation of the inner water droplets the channel wall must be hydrophobic 
whereas the production of the outer oil droplets demands hydrophilic surface properties. Hence, the surface energy of the microchannel has to be patterned. Abate and Weitz ${ }^{25}$ used photolithographic techniques to realize alternating wettability profiles inside their microchannels and produced multiple emulsion with high precision.

We followed another approach and used our simple and convenient LbL deposition technique to selectively apply a hydrophilic channel coating in certain areas of the microfluidic device (Fig. 4). In Fig. 4(a) a schematic diagram of the chip capable of forming double emulsions is presented. The microchannel comprises an upper, narrow part (50 $\mu \mathrm{m}$ wide channels) and a lower, wider part (200 $\mu \mathrm{m}$ wide channels). Those sections meet at the second flow-focusing region, where the enclosure of the small water in the bigger oil droplets and hence the wettability reversal has to occur. In order to produce stable w/o/w double emulsions the lower part must be turned hydrophilic selectively over the lower one. Directly after device assembly we injected a polyelectrolyte sequence containing FITC labeled PAH in inlet D and flushed it through the lower part of the chip at a constant flow rate of $50 \mu \mathrm{L} \mathrm{h}^{-1}$. Simultaneously we blocked the upper part of the device by streaming DIW through inlet B at $100 \mu \mathrm{L} \mathrm{h}^{-1}$. Inlet $\mathrm{C}$ was closed during the modification process while $\mathrm{A}$ was used as an outlet.

By means of fluorescence microscopy we analyzed the microfluidic device after this hydrophilic treatment. Fig. 4(b) and (c)

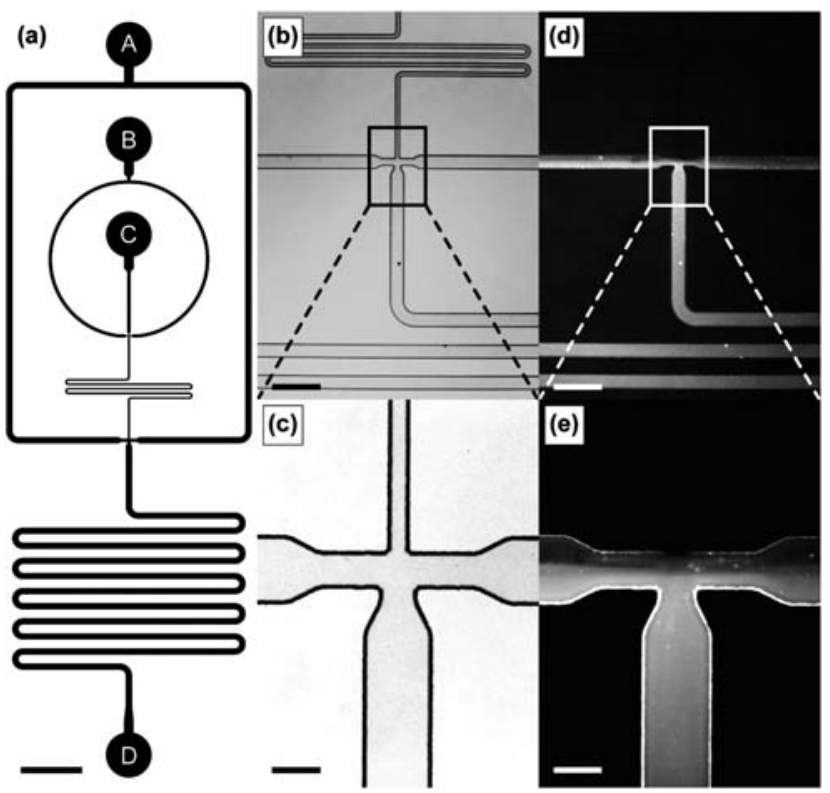

Fig. 4 Selective hydrophilic surface coating of certain parts of the microchannel. (a) Schematic of the microfluidic device used for the generation of w/o/w double emulsions. For hydrophilic surface treatment a polyelectrolyte sequence was flushed through inlet D, while DIW was injected through inlet B. Inlet $\mathrm{C}$ was blocked and A was used as an outlet. (b and c) Bright field micrographs of the microfluidic device after hydrophilic surface treatment. ( $\mathrm{d}$ and e) Corresponding fluorescence micrographs of the microfluidic device after hydrophilic surface treatment. Only the lower part of the microchannel was coated with a fluorescent PEM. No deposition occurred within the upper part. Scale bars denote (a) $2 \mathrm{~mm}$, (b) $750 \mu \mathrm{m}$, (c) $150 \mu \mathrm{m}$, (d) $750 \mu \mathrm{m}$ and (e) $150 \mu \mathrm{m}$. show bright field micrographs of the area around the second flow-focusing region. The corresponding fluorescence micrographs in Fig. 4(d) and (e) reveal that only in the designated lower part of the microchannel a fluorescent PAH-PSS-PAHPSS PEM was deposited. In contrast, the absence of a fluorescence signal in the upper part of the chip proves that no LbL coating took place there. The boundary between the modified and the non-modified channel section can be located exactly at the second flow-focusing region. These results demonstrate that following our LbL surface modification method we are able to partially coat the microchannel wall with a hydrophilic PEM in a precise and spatially defined manner. No lithographic tools are needed, a flow pattern is transferred instead. It should be pointed out that our partial surface modification technique is very robust for the geometries tested here, but more complicated channel designs, e.g. chips with multiple flow-focusing junctions, might prove challenging.

In a second surface modification step we produced a defined hydrophobic surface in the upper part of the microchannel. Again we used flow patterning to achieve a partial coating. In a reversed setup compared to the hydrophilic modification step we slowly injected the commercial water repellent agent Aquapel through inlet B at $20 \mu \mathrm{L} \mathrm{h}^{-1}$. A stream of air was blown in the channel through inlet $\mathrm{D}$ at $5000 \mu \mathrm{L} \mathrm{h}^{-1}$ to prevent Aquapel from interfering with the PEM deposited in the lower part of the chip. While inlet $\mathrm{C}$ was blocked during this process $\mathrm{A}$ was used as an outlet.

Subsequently, we used the resultant partially hydrophilic, partially hydrophobic device for the production of a w/o/w double emulsion (Fig. 5). DIW containing $0.5 \%$ w/w SDS was injected as the outermost phase through inlet $\mathrm{A}$ at $1500 \mu \mathrm{L} \mathrm{h}^{-1}$. Through inlet $\mathrm{B}$ we flushed the fluorous oil FC-40 containing $2.0 \% \mathrm{w} / \mathrm{w}$ EA surfactant ${ }^{26}$ at a flow rate of $200 \mu \mathrm{L} \mathrm{h}^{-1}$. Pure DIW was used as the innermost phase and applied through inlet $\mathrm{C}$ at $150 \mu \mathrm{L} \mathrm{h}^{-1}$. In Fig. 5(a) and (b) the formation of the double emulsion is depicted. DIW-in-oil droplets formed at the first flow-focusing region travel smoothly through the upper part of the channel. The microchannel wall is wetted by the fluorous oil indicating that the Aquapel treatment was successful. At the second flow-focusing region every DIW droplet is enclosed in a larger oil droplet. No oil droplet stays empty since both droplet formation steps proceed at exactly the same frequency of $130 \mathrm{~Hz}$. The double emulsion droplets proceed through the lower part of the microchannel without any wetting problems. During the whole observation period of $90 \mathrm{~min}$ no signs of degradation in hydrophilic surface properties occurred.

The resultant double emulsion was extruded through outlet $\mathrm{D}$ and collected for $30 \mathrm{~min}$. A small fraction of $c a .5 \mu \mathrm{L}$ was injected into a storage device. Fig. 5(c) shows a micrograph of the hexagonal close-packed monolayer adopted by the double emulsion droplets inside the reservoir. The droplet diameter distribution of 1107 droplets, shown in Fig. 5(d), is extremely narrow for both the inner water and the outer oil droplets and yields mean values of $84.6 \mu \mathrm{m}$ and $109.4 \mu \mathrm{m}$, respectively. The standard deviations, $0.8 \mu \mathrm{m}$ for the inner and $1.0 \mu \mathrm{m}$ for the outer droplets, are below $1 \%$ of the corresponding average diameter, proving the high level of monodispersity in both cases.

In this experimental study we showed that our LbL surface deposition technique is not limited to simply turning a complete 

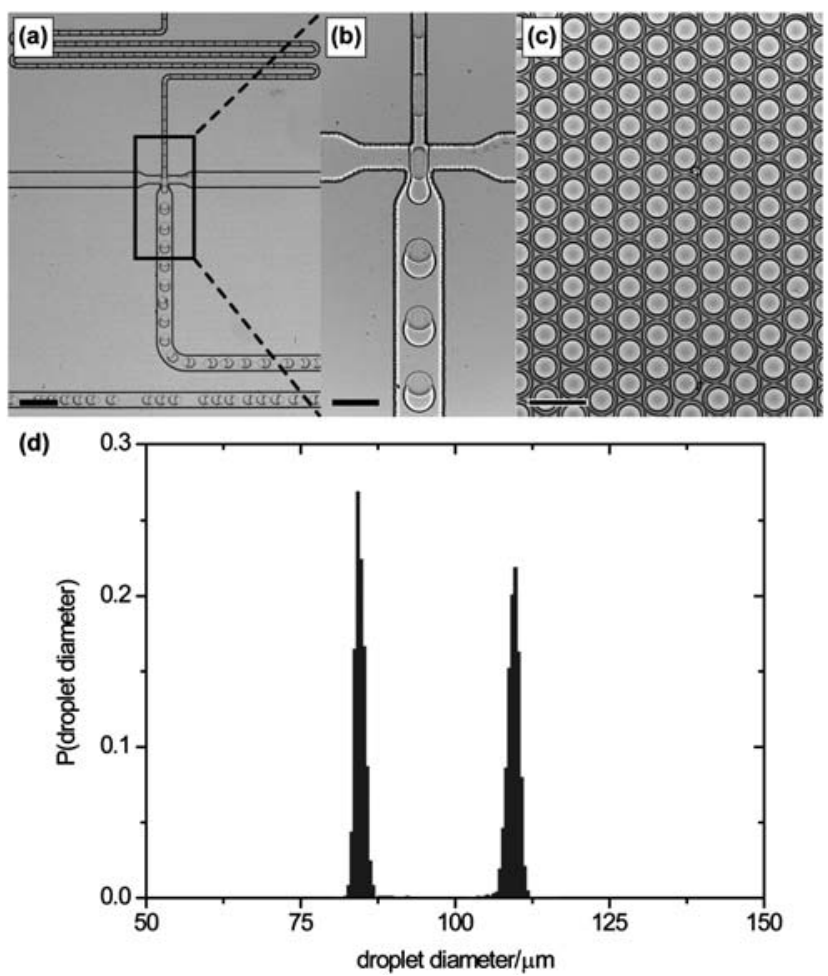

Fig. 5 Production of a w/o/w double emulsion using a partially hydrophilic, partially hydrophobic device. (a and b) Micrographs of the stable enclosing of the small water droplets in the bigger oil droplets. (c) This micrograph depicts the hexagonal close-packed monolayer adopted by the $\mathrm{w} / \mathrm{o} / \mathrm{w}$ emulsion in a storage device. (d) The diameter distribution shows a high level of droplet monodispersity both for the inner and the outer droplets. Scale bars denote (a) $500 \mu \mathrm{m}$, (b) $200 \mu \mathrm{m}$ and (c) $200 \mu \mathrm{m}$.

microchannel hydrophilic. In contrast, it is possible to precisely pattern the surface energy within assembled channels. One application is the fabrication of semi-hydrophilic, semi-hydrophobic microfluidic devices capable of producing highly monodisperse $\mathrm{w} / \mathrm{o} / \mathrm{w}$ double emulsions.

\section{Experimental}

\section{Device fabrication}

Microfluidic devices were fabricated by conventional soft lithographic techniques. ${ }^{2}$ Different microchannel architectures were designed with AutoCAD (AutoDesk) and transferred to highresolution photomasks fabricated on transparencies (Circuit Graphics). The negative photoresist SU-8 2025 (MicroChem) was spin-coated onto 3 inch silicon wafers (Compart Technology) and patterned using a MJB4 mask aligner (Süss MicroTec). Development was accomplished by immersion into 1-methoxy-2-propyl acetate (Sigma-Aldrich).

A commercially available Sylgard 184 PDMS kit (Dow Corning), containing the pre-polymer and a cross-linker, was used in the recommended ratio of $10: 1 \mathrm{w} / \mathrm{w}$. The mixture was poured on top of the patterned silicon wafers and degassed. After curing at $80^{\circ} \mathrm{C}$ for $10 \mathrm{~h}$ the PDMS cast was cut and peeled off the wafers. Inlets and outlets were stamped out using a biopsy punch (Kai Industries) with an outer diameter of $1 \mathrm{~mm}$. The microfluidic devices were assembled by joining the PDMS cast and either a glass slide or a flat sheet of cured PDMS stripped off a blank wafer. Bonding strength was provided by pre-treating both contact surfaces with oxygen plasma for $8 \mathrm{~s}$ in a Femto plasma cleaner (Diener electronic).

Storage devices were fabricated following the same process. Instead of patterned wafers microscope cover slips glued onto glass slides were used as casting molds.

\section{Surface modification}

Hydrophilic channel coatings were produced by LbL PEM deposition onto the microchannel surface directly after device assembly. Solutions of $\mathrm{NaCl}$ (AnalaR) in DIW $(0.1 \mathrm{M})$ as well as of PAH ( $M_{\mathrm{w}} \approx 56000$, Sigma-Aldrich) and PSS $\left(M_{\mathrm{w}} \approx 70000\right.$, Sigma-Aldrich), both $0.1 \% \mathrm{w} / \mathrm{v}$ in $0.5 \mathrm{M}$ aqueous $\mathrm{NaCl}$ solution, were prepared. In the case of subsequent fluorescence measurements PAH labelled with FITC fluorescence dye $\left(M_{\mathrm{w}} \approx 56000\right.$, $\mathrm{PAH}:$ FITC $\approx 50: 1$, Sigma-Aldrich) was used instead of pure PAH. A piece of PE tubing (Becton Dickinson) was loaded with a sequence of $5 \mathrm{~cm}$ long polyelectrolyte segments (alternatingly PAH and PSS) with $2.5 \mathrm{~cm}$ long $\mathrm{NaCl}$ segments in between. All solution segments were separated from each other by $1 \mathrm{~cm}$ long air plugs. Using a PHD 2000 syringe pump (Harvard Apparatus) the whole sequence was sequentially flushed through the respective microchannel at a constant flow rate of $50 \mu \mathrm{L} \mathrm{h}^{-1}$. For a partial hydrophilic surface modification the LbL coating of certain areas of a microchannel was prevented by simultaneous injection of DIW into the device through a suitable inlet at a flow rate of $100 \mu \mathrm{L} \mathrm{h}^{-1}$.

A partial hydrophobic surface treatment was accomplished by slowly introducing the commercially available water repellent agent Aquapel (PPG Industries) into the assembled microchannel at $20 \mu \mathrm{L} \mathrm{h}^{-1}$. Channel regions which should not be coated were blocked by an air stream with a flow rate of $5000 \mu \mathrm{L} \mathrm{h}^{-1}$. After filling the designated part of the microchannel with Aquapel it was blown out again by the air stream.

\section{Microfluidic experiments}

In o/w droplet formation experiments hexadecane (Fluka) was used as dispersed phase and DIW containing $0.5 \% \mathrm{w} / \mathrm{w}$ SDS (Sigma-Aldrich) as continuous phase. For w/o/w double emulsions DIW was applied as the innermost phase, FC-40 (SigmaAldrich) containing $2.0 \% \mathrm{w} / \mathrm{w}$ of EA surfactant ${ }^{26}$ as the middle phase and DIW containing $0.5 \% \mathrm{w} / \mathrm{w}$ SDS as the outermost phase.

All liquids were injected into microfluidic devices via $\mathrm{PE}$ tubes (Becton Dickinson). For high-throughput o/w droplet formation experiments the tubing was glued into the device. A $5: 1 \mathrm{w} / \mathrm{w}$ mixture of PDMS pre-polymer and its cross-linker (Dow Corning) was pre-cured for $10 \mathrm{~min}$ at $80^{\circ} \mathrm{C}$, applied and completely cured at $\mathrm{rt}$ for one day. In all microfluidic experiments PHD 2000 syringe pumps (Harvard Apparatus) were used to inject liquids at constant flow rates between $50 \mu \mathrm{L} \mathrm{h}^{-1}$ and $4500 \mu \mathrm{L} \mathrm{h}^{-1}$.

\section{Data acquisition and analysis}

Droplet formation on-chip as well as emulsion samples inside storage devices were imaged using a monochrome Phantom v7.2 
camera (Vision Research) attached to an IX71 inverted microscope (Olympus). The frequency of droplet formation and the droplet diameter distribution were calculated using LabVIEW 8.2 (National Instruments).

Fluorescence micrographs were obtained using a blue LED (Thorlabs) emitting at a wavelength of $470 \mathrm{~nm}$ as a light source and an EMCCD iXonEM+ DU 897 camera (Andor Technology) coupled with an IX71 inverted optical microscope (Olympus) for detection.

\section{Conclusions}

In summary, we developed a largely automated LbL surface modification method yielding hydrophilic microchannels in PDMS-based devices, which outperforms existing techniques in terms of rapidness and convenience. We are able to produce long-term stable hydrophilic coatings allowing for the production of monodisperse o/w droplets even months after the modification step. Furthermore, we successfully used our method for high-throughput droplet generation at flow rates of several $\mathrm{mL}$ $\mathrm{h}^{-1}$. By introducing a wide cavity into the microchannel we regularized the jet breakup and obtained highly monodisperse o/w droplets. We also used our LbL deposition technique to transfer a flow pattern onto the microchannel surface. In combination with a subsequent hydrophobic surface treatment step we obtained partially hydrophilic, partially hydrophobic microfluidic devices which we used for the production of monodisperse $\mathrm{w} / \mathrm{o} / \mathrm{w}$ double emulsions.

\section{Acknowledgements}

This work was supported by the EPSRC, the RCUK Basic Technology Programme, Fonds der Chemischen Industrie and the Cambridge European Trusts. We thank Raindance for generously providing EA surfactant.

\section{Notes and references}

1 J. C. McDonald, D. C. Duffy, J. R. Anderson, D. T. Chiu, H. Wu, O. J. A. Schueller and G. M. Whitesides, Electrophoresis, 2000, 21, 27; J. C. McDonald and G. M. Whitesides, Acc. Chem. Res., 2002, 35, 491.

2 D. Qin, Y. Xia and G. M. Whitesides, Adv. Mater., 1996, 8, 917; D. C. Duffy, J. C. McDonald, O. J. A. Schuller and G. M. Whitesides, Anal. Chem., 1998, 70, 4974.

3 H. Makamba, J. H. Kim, K. Lim, N. Park and J. H. Hahn, Electrophoresis, 2003, 24, 3607; I. Wong and C.-M. Ho, Microfluid. Nanofluid., 2009, 7, 291.

4 M. L. van Poll, F. Zhou, M. Ramstedt, L. Hu and W. T. S. Huck, Angew. Chem., Int. Ed., 2007, 46, 6634; M. L. van Poll, S. Khodabakhsh, P. J. Brewer, A. G. Shard, M. Ramstedt and W. T. S. Huck, Soft Matter, 2009, 5, 2286.

5 G. Ocvirk, M. Munroe, T. Tang, R. Oleschuk, K. Westra and D. J. Harrison, Electrophoresis, 2000, 27, 107; Y. Dou, N. Bao, J. Xu and H. Chen, Electrophoresis, 2002, 23, 3558; J. H. Xu, G. S. Luo, S. W. Li and G. G. Chen, Lab Chip, 2006, 6, 131.

6 M. J. Owen and P. J. Smith, J. Adhesion Sci. Technol., 1994, 8, 1063; Y. Lai, Y. Y. Lin, Y. L. Denq, S. S. Shyu and J. K. Chen, J. Adhesion Sci. Technol., 1996, 10, 231.

7 J. Kim, M. K. Chaudhury and M. J. Owen, J. Colloid Interface Sci., 2000, 226, 231; H. Hillborg, N. Tomczak, A. Olàh, H. Schönherr and
G. J. Vancso, Langmuir, 2004, 20, 785; A. Olàh, H. Hillborg, H. Schönherr and G. J. Vancso, Appl. Surf. Sci., 2005, 239, 410.

8 M. Morra, E. Occhiello, R. Marola, F. Garbassi, P. Humphrey and D. Johnson, J. Colloid Interface Sci., 1990, 137, 11; E. P. Everaert, H. C. van der Mei and H. J. Busscher, J. Adhesion Sci. Technol., 1996, 10, 351

9 J. Lahann, M. Balcells, H. Lu, T. Rodon, K. F. Jensen and R. Langer, Anal. Chem., 2003, 75, 2117; H.-Y. Chen, A. A. McClelland, Z. Chen and J. Lahann, Anal. Chem., 2008, 80, 4119.

10 N. Patrito, C. McCague, S. Chiang, P. R. Norton and N. O. Petersen, Langmuir, 2006, 22, 3453; D. Wu, B. Zhao, Z. Dai, J. Qina and B. Lin, Lab Chip, 2006, 6, 942; L. M. Fidalgo, C. Abell and W. T. S. Huck, Lab Chip, 2007, 7, 984.

11 S. Hu, X. Ren, M. Bachman, C. E. Sims, G. P. Li and N. L. Allbritton, Anal. Chem., 2004, 76, 1865; Y. Wang, H.-H. Lai, M. Bachman, C. E. Sims, G. P. Li and N. L. Allbritton, Anal. Chem., 2005, 77, 7539.

12 A. R. Abate, D. Lee, T. Do, C. Holtze and D. A. Weitz, Lab Chip, $2008,8,516$

13 G. Decher, J. D. Hong and J. Schmitt, Thin Solid Films, 1992, 210/ 211, 831; G. Decher, Y. Lvov and J. Schmitt, Thin Solid Films, 1994, 244, 772; G. Decher, Science, 1997, 277, 1232.

14 H. Katayama, Y. Ishihama and N. Asakawa, Anal. Chem., 1998, 70, 5272; Y. Liu, J. C. Fanguy, J. M. Bledsoe and C. S. Henry, Anal. Chem., 2000, 72, 5939; K. W. Ro, W.-J. Chang, H. Kim, Y.-M. Koo and J. H. Hahn, Electrophoresis, 2003, 24, 3253.

15 D. L. Chen and R. F. Ismagilov, Curr. Opin. Chem. Biol., 2006, 10, 226; B. Zheng and R. F. Ismagilov, Angew. Chem., Int. Ed., 2005, 44, 2520; T. Hatakeyama, D. L. Chen and R. F. Ismagilov, J. Am. Chem. Soc., 2006, 128, 2518; J. Pei, Q. Li, M. S. Lee, G. A. Valaskovic and R. T. Kennedy, Anal. Chem., 2009, 81, 6558.

16 S. L. Anna, N. Bontoux and H. A. Stone, Appl. Phys. Lett., 2003, 82, 364.

17 S. L. Anna and H. C. Mayer, Phys. Fluids, 2006, 18, 121512; A. S. Utada, A. Fernandez-Nieves, H. A. Stone and D. A. Weitz, Phys. Rev. Lett., 2007, 99, 094502; P. Guillot, A. Colin, A. S. Utada and A. Ajdari, Phys. Rev. Lett., 2007, 99, 104502; M. De Menech, P. Garstecki, F. Jousse and H. A. Stone, J. Fluid Mech., 2008, 595, 141; P. Guillot, A. Colin and A. Ajdari, Phys. Rev. E: Stat. Phys., Plasmas, Fluids, Relat. Interdiscip. Top., 2008, 78, 016307; P. Guillot, A. Ajdari, J. Goyon, M. Joanicot and A. Colin, C. R. Chim., 2009, 12, 247.

18 L. Rayleigh, Proc. London Math. Soc., 1878, s1-10, 4; L. Rayleigh, Proc. R. Soc. London, 1879, 29, 71.

19 A. Clarke, Eastman Kodak Company, WO Pat., 004314 A1, 2009.

20 Z. Nie, S. Xu, M. Seo, P. C. Lewis and E. Kumacheva, J. Am. Chem. Soc., 2005, 127, 8058; S. Xu, Z. Nie, M. Seo, P. Lewis, E. Kumacheva, H. A. Stone, P. Garstecki, D. B. Weibel, I. Gitlin and G. M. Whitesides, Angew. Chem., Int. Ed., 2005, 44, 724; T. Nisisako and T. Torii, Adv. Mater., 2007, 19, 1489; W. Li, H. H. Pham, Z. Nie, B. MacDonald, A. Guenther and E. Kumacheva, J. Am. Chem. Soc., 2008, 130, 9935; S. Dubinsky, H. Zhang, Z. Nie, I. Gourevich, D. Voicu, M. Deetz and E. Kumacheva, Macromolecules, 2008, 41, 3555; Q. Xu, M. Hashimoto, T. T. Dang, T. Hoare, D. S. Kohane, G. M. Whitesides, R. Langer and D. G. Anderson, Small, 2009, 5, 1575.

21 W. Seifriz, J. Phys. Chem., 1925, 29, 738; A. T. Florence and D. Whitehill, Int. J. Pharm., 1982, 11, 277; N. Garti, Colloids Surf., A, 1997, 123-124, 233.

22 T. Nisisako, Chem. Eng. Technol., 2008, 31, 1091.

23 S. S. Davis and I. M. Walker, Methods Enzymol., 1987, 149, 51; F. Cournarie, M.-P. Savelli, V. Rosilio, F. Bretez, C. Vauthier, J.-L. Grossiord and M. Seiller, Eur. J. Pharm. Biopharm., 2004, 58, 477.

24 S. Matsumoto, Y. Kita and D. Yonezawa, J. Colloid Interface Sci., 1976, 57, 353; G. T. Vladisavljević, M. Shimizu and T. Nakashima, J. Membr. Sci., 2006, 284, 373.

25 A. R. Abate and D. A. Weitz, Small, 2009, 5, 2030.

26 C. Holtze, A. C. Rowat, J. J. Agresti, J. B. Hutchison, F. E. Angilè, C. H. J. Schmitz, S. Köster, H. Duan, K. J. Humphry, R. A. Scanga, J. S. Johnson, D. Pisignano and D. A. Weitz, Lab Chip, 2008, 8, 1632. 